\title{
LA BANCA ISLÁMICA: SITUACION ACTUAL Y RETOS
}

\section{ISLAMIC BANKING: CURRENT SITUATION AND CHALLENGES}

\section{AUTORA}

\section{Pilar Sánchez González}

Facultad de Ciencias Económicas y Empresariales. Universidad Complutense de Madrid. gamonpumareta@hotmail.com

\section{RESUMEN}

El presente trabajo pretende analizar cuál es la situación actual de la Banca Islámica y cuáles son los retos a los que se enfrenta. Para ello, analizaremos en primer lugar qué es la Banca Ética, puesto que la Banca Islámica se encuentra en esta tipología. A continuación, se tratará la Banca Islámica en profundidad y por último, veremos cuáles son los retos a los que se enfrenta.

\section{PALABRAS CLAVE}

Banca Islámica - Banca Ética - Marketing.

\section{ABSTRACT}

This study attempts to analyze what is the current state of Islamic banking and what are the challenges it faces. We analyze first what is the Ethical Banking, since the Islamic Banking is in this typology. Then, it will be Islamic Banking in depth and finally, I will discuss the challenges it faces. 


\section{KEY WORDS}

Islamic Banking - Ethical Banking - Marketing.

\section{ÍNDICE}

1. Introducción.

2. De la Banca Tradicional a la Banca Ética.

3. Banca Islámica.

4. Tipos de contratos.

5. Estructura de la institución bancaria islámica.

6. Experiencia internacional de la Banca Islámica en algunos países.

6.1. Pakistán.

6.2. Irán.

6.3. Sudán.

6.4. Bahrein.

6.5. Malasia.

6.6. Arabia Saudí.

7. Situación actual y retos.

8. Bibliografía.

9. Webgrafía.

\section{Introducción}

En cualquier economía, existe la necesidad de transferir fondos desde los ahorradores a los inversores, ya que las personas que ahorran no son a menudo aquellas que tienen la capacidad de explotar las ocasiones de inversión rentables. Esta función se realiza a través del proceso de intermediación financiera. Los 
intermediarios financieros están en mejor posición para recopilar información acerca de las oportunidades de inversión, lo que es crucial para una asignación eficiente de los recursos de la sociedad.

Las funciones que los bancos realizan son importantes tanto si la economía en cuestión es laica como si es islámica. Las personas necesitan los servicios bancarios. Sin embargo, los bancos tradicionales realizan sus actividades de empréstito y préstamo, y la mayoría del resto de funciones, sobre la base de un interés fijo. En una economía islámica está prohibido tanto ofrecer como aceptar interés.

Aunque los bancos islámicos surgieron en respuesta a las necesidades de mercado de los clientes musulmanes, no son instituciones religiosas. Como otros bancos, son instituciones que persiguen el beneficio.

\section{De la Banca Tradicional a la Banca Ética}

Banca Ética es un nombre polémico porque deja entender implícitamente que el resto de bancos no son éticos. Además, se argumenta que la ética es un concepto difícil de definir, al estar influido por valoraciones subjetivas que pueden evolucionar y variar de un país a otro. Por estos motivos, algunas personas prefieren hablar de Banca Social o Banca Alternativa. Nuestro tratamiento será de Banca Ética, pero dejando claro que no estamos definiendo a la Banca Tradicional como "no ética".

Desde hace unos años, la Banca Ética es un tema de actualidad. La concesión del premio Nobel de la Paz a Muhammad Yunus, fundador del Graneen Bank, fue la demostración, a nivel internacional, de la preocupación que el mundo tiene sobre un nuevo tipo de Banca. En el caso de Europa, y desde la década de los 80, existen entidades como Triodos Bank (Holanda), Okiobank (Alemania) o ABS (Suiza), preocupadas no sólo por el valor monetario del dinero, sino también por el valor social del mismo. 
Después de un parón significativo que duro más de una década, con el inicio del nuevo siglo, resurgió con fuerza el interés de desarrollar un banco ético en España. Entre las múltiples iniciativas, podemos señalar la creación de la Red de Útiles Financieros (RUFAS), la Fundación Solidaridad Económica (REAS Euskalerria), la Asociación por la Banca Ética y Solidaria, FETS (Financament Etic i Solidari), el Proyecto Trust y FIARE, provocando, estos dos últimos proyectos, la implantación de Triodos Bank y la Banca Ética I taliana, respectivamente.

En cuanto a la conceptualización la Banca Ética, se autodefine, frente a la Banca Tradicional, a la que califican de técnica y anónima, como instituciones que se diferencian, por los sectores o tipos de actividades en las que depositan el dinero (activo) y por la opción de crear conciencia y compromiso entre los depositarios, permitiéndoles decidir el destino de sus fondos (pasivo).

En este contexto, el objetivo de este capítulo es establecer las diferencias más significativas entre los llamados Bancos Tradicionales (Bancos, Cajas de Ahorros y Cooperativas de Crédito) y la Banca Ética. Para ello, trataremos la Responsabilidad Social Corporativa, incorporada actualmente en muchas de las grandes compañías del mundo y que abarca una serie de conceptos vinculados con la ética o el desenvolvimiento ético de las organizaciones. En el sector bancario, bajo este término, se incluyen de forma indiferente conceptos como el de Etica Bancaria, Fondos Éticos, Inversión Solidaria, Responsabilidad Social y, por último, el que nos ocupa, Banca Ética.

Las "Inversiones Socialmente Responsables" tienen su origen en la década de los 50 en Estados Unidos y se vincularon principalmente al rechazo que ciertos grupos religiosos como los Cuáqueros promovían con respecto a que su dinero se utilizara en "inversiones pecaminosas", como el juego, el alcohol o el tabaco. Posteriormente, en la década de los 60 , este movimiento adquiere un carácter reivindicativo a través 
de acciones en contra de la guerra de Vietnam o en los 80 , en contra del régimen de Sudáfrica. A finales del siglo pasado, estas actuaciones se generalizaron a ámbitos más empresariales, oponiéndose a actividades de contaminación ecológica, que efectuaran experimentación con animales, la explotación infantil o las relaciones comerciales injustas. Dicho "movimiento" supuso, en su vertiente más pragmática, la aparición de fondos de inversión responsables, y en un aspecto más teórico, el desarrollo del concepto de "Responsabilidad Social Corporativa".

La Inversión Socialmente Responsable como concreción de la Responsabilidad Social Corporativa se puede definir como aquella que combina los criterios éticos con los económicos, de forma que las inversiones no tengan en cuenta sólo las condiciones financieras, como rentabilidad o seguridad, sino también la utilización de los fondos por parte de las entidades gestoras. Estas inversiones, siguiendo a Alsina (2002), se realizarán de acuerdo con los criterios, consecuentes con los de las personas depositarias de los fondos. Dichos criterios podrán ser negativos o positivos. La utilización del criterio "negativo", da lugar a vetar la inversión en empresas que desarrollen productos o servicios relacionados con algunos de los siguientes temas: armamento, tabaco, alcohol, pornografía, juego, ejército, explotación laboral, contaminación, manipulación genética, experimentación animal, energía nuclear, tala de bosques, minería contaminante, deslocalización, manipulación de consumidores, diferencias salariales, apoyo a partidos políticos o regímenes dictatoriales, especulación financiera, evasión de impuestos, drogas y mafia.

En el caso de los criterios éticos "positivos", las inversiones se colocarán en empresas que tengan por objeto la fabricación de productos que generen valor social, la utilización de energías medioambientales, se dediquen al reciclaje, den apoyo a la biodiversidad y al desarrollo local, se fundamenten en la igualdad de oportunidades, valoren la relación con los stakeholders (son aquellas personas, entidades o grupos, tanto formales como informales, que tienen intereses legítimos en el funcionamiento de una empresa, esto incluye a directivos, trabajadores, accionistas, clientes, 
proveedores, vecinos... o cualquier otro que de forma activa o pasiva se vea afectado por el funcionamiento de una determinada empresa), apoyen la formación, se relacionen con sindicatos, ofrezcan transparencia, apoyen el consumo justo y responsable, ofrezcan transferencia tecnológica al Sur o respeten los derechos humanos, entre otros.

Además, hay que señalar que la inversión Socialmente Responsable se efectúa a través de los fondos, pudiendo recibir la denominación de éticos o solidarios en función de si establecen filtros éticos o ceden parte de los beneficios para causas sociales, respectivamente. La inversión en estos fondos puede generar una rentabilidad financiera similar a las inversiones en el mercado tradicional, así lo indican los diversos trabajos realizados al respecto en diferentes países. Por un lado, el informe Social Investment Forum (1999), señala que el 70 por ciento de los fondos éticos en Estados Unidos obtuvo las rentabilidades más altas posibles, y que la mitad llegaron a ser primeros en las clasificaciones de fondos.

Por otro lado, un estudio del Ethical Investment Research Service (EIRIS) en el que se analizan los resultados de 15 fondos británicos, demuestra que estos fondos, de media, tienen menos riesgo que los fondos que carecen de criterios éticos, aunque en términos medios tienen rendimientos también más bajos, lo cual parece lógico si tenemos en cuenta que el riesgo y la rentabilidad se mueven en la misma dirección y que en muchos de estos fondos se generan más gastos motivados por el análisis ético de la cartera. No obstante, tal como tratan De la Cuesta y del Río (2001), el mayor fondo de pensiones británico, el Friends Provident Stewardship Fund, obtuvo en el periodo 1990-1998 una tasa de crecimiento del 21,4 por ciento, frente a una media del 15 por ciento en el sector. En el caso español, algunos de los bancos que tienen Fondos de Inversión Éticos (FIE) son: Grupo Santander, BBVA, Caja Madrid, La Caixa y la BBK. 
Con el objetivo de definir qué es un Banco Ético, se han consultado las definiciones de diferentes autores (Green, 1989; Lynch, 1991; Cowton, 1999; Alsina, 2002; Ballesteros, 2003, Kendric, 2004; De la Cuesta, 2006; Sasía, 2008, entre otros) sobre este término. La mayoría coinciden en considerar dos dimensiones simultáneas, la financiación de actividades económicas con un impacto social positivo, la primera, y la obtención de beneficios, la segunda. Un claro ejemplo es la filosofía mantenida por Triodos Bank, inspirada en las tres P's: Planet (planeta), People (personal) y Profit (beneficios).

Desde esta perspectiva, podríamos considerar que las características necesarias para poder definir la Banca Ética son las señaladas por De la Cuesta y Del Río (2001) tienen que disponer de:

- La máxima participación social, sin que eso signifique la obligatoriedad de renunciar a la posibilidad de delegación basada en la confianza. La participación social se traduce sobre todo en la posibilidad de seleccionar el destino de las inversiones en función no sólo de criterios de rentabilidad, sino también en función de las inquietudes sociales de los ahorradores e inversores.

- La gestión eficaz y profesional de la actividad empresarial que supone el nuevo instrumento financiero.

- La transparencia como valor fundamental, en la gestión administrativa y los procesos de toma de decisiones, y especialmente en la aplicación de fondos.

- La existencia de un Código Ético explícito que rija el proceso de toma de decisiones, y a partir del cual se pueden generar informes éticos o de beneficio social, que acompañen en igualdad a los informes técnicos y financieros de los proyectos o empresas en el momento de decidir sobre su posible financiación.

Además, San J osé y Retozala (2006) añaden: 
- La colocación del activo en proyectos con valor añadido social y en ningún caso en proyectos especulativos o que incumplan directamente los criterios negativos señalados con anterioridad en referencia a los fondos de inversiones.

- El carácter global de la característica anterior, en el sentido de que no incumba sólo a una parte de la actividad bancaria, sino a la totalidad de la misma y de las posibles entidades en las que ésta pudiera participar, o estar participada de forma significativa. Es decir, un banco que nos ofrezca la garantía total de que todo nuestro dinero no está apoyando financieramente todas aquellas prácticas empresariales que quisiéramos ver transformadas 0 sustituida por otras más acordes con un modelo de sociedad y de economía solidaria.

En 2006 y tomando como fuente "Social Investment Forum", "European SRI Study 2006, Washington", se estimaba que existían productos relacionados con la inversión socialmente responsable en 21 países, un total de 650 fondos de inversión éticos en el mundo: 375 en Estados Unidos( con un valor de 179.000 millones de dólares), 375 fondos en Europa (con un valor de 24.000 millones de euros), 53 fondos en Canadá (con 18.000 millones de dólares) e inversiones menores en Australia y J apón (11.000 y 2.000 millones de dólares respectivamente).

Un ejemplo de la Banca Ética es la Banca Islámica, aunque existen algunas diferencias, siguiendo a De Anca, son tres: los criterios de inversión que utilizan, los órganos de supervisión con los que controlan sus operaciones y la base de clientes sobre la que se apoyan.

La mayoría de los fondos islámicos, al igual que los éticos, cuentan con órganos de supervisión: Consejos de Sharia en el caso Islámico y Comités Éticos en el de los fondos de Inversión Socialmente Responsable. Estos órganos difieren en su 
funcionamiento, ya que los Comités de Sharia incluyen expertos en Derecho Islámico que deciden la legalidad de operaciones financieras concretas según los principios de la jurisprudencia islámica, mientras que los integrantes de las Comisiones Éticas son académicos, miembros de ONGs o empresarios y su labor no atiende tanto a definir la legalidad de las operaciones financieras sino que se centra, por un lado, en diseñar los criterios de inversión del fondo, de acuerdo con la base de sus clientes, y, por otro, en asegurar, frente a los gestores financieros, que estos criterios se cumplen.

\section{Banca Islámica}

Cuando surgió la banca comercial, después de la revolución industrial, un gran número de musulmanes se abstuvo de tratar con bancos comerciales. Sin embargo, las necesidades crecientes de los comerciantes, industriales y empresarios ejercieron una gran presión para que las economías se monetizaran con rapidez. Los economistas y bancos musulmanes aceptaron el reto de desarrollar modelos alternativos de intermediación financiera. Un valioso trabajo teórico se desarrolló a principios del siglo XIX. En aquel entonces, la mayor parte del mundo musulmán estaba bajo dominio colonial. Cuando los países musulmanes obtuvieron su independencia, después de la Segunda Guerra Mundial, empezaron a desarrollarse experimentos prácticos en la financiación sin intereses, a escala modesta, ampliando su alcance gradualmente.

Entre los musulmanes ha existido siempre, incluso en la época colonial, una demanda de productos financieros adaptados a su credo y conformes a los principios de la legislación islámica, la Sharia. Para Abuamria (2006), el primer experimento moderno de banco islámico se realizó en el año 1963, en Egipto, de forma encubierta, sin proyectar una imagen islámica, por miedo a ser visto como una manifestación de fundamentalismo islámico. Sin embargo, los primeros movimientos para establecer bancos islámicos se produjeron en los años cuarenta en Malasia y en los cincuenta en Pakistán. 
En los años setenta, hubo cambios en el panorama político de muchos países islámicos, por lo que ya no era necesario crear instituciones financieras islámicas encubiertas. La primera institución libre de intereses que incorporó en su nombre la palabra "banco", el Nasser Social Bank, se estableció también en Egipto, en 1971. Ésta fue, según Iqbal (2006), la primera vez que un gobierno de un país musulmán mostraba interés en incorporar una institución libre de intereses. Si bien los objetivos del Nasser Social Bank eran principalmente sociales, como el suministro de préstamos sin intereses a personas pobres y necesitadas, becas a estudiantes y microcréditos para pequeños proyectos sobre la base del reparto de los beneficios, la participación de una autoridad pública en la banca sin intereses envió señales importantes a los empresarios musulmanes que tenían exceso de fondos. Un grupo de estos empresarios tomó la iniciativa de establecer el Dubai I slamic Bank en 1975, en Dubai, Emiratos Árabes Unidos (EAU). Este fue el primer banco islámico establecido a través de la iniciativa privada. Sin embargo, el apoyo oficial fue crucial por parte de los gobiernos de EAU y Kuwait, contribuyendo respectivamente con el 20 y el 10 por 100 del capital.

En 1974, y por acuerdo de cuarenta países musulmanes, se creó el Banco Islámico para el Desarrollo, con el objetivo de promover la cooperación económica bajo los principios islámicos, como el de la equidad social, que entre otros, es la base de su filosofía respecto a la participación en los resultados de las inversiones financieras. Este principio de equidad se concreta en la concesión de financiación gratuita para los más necesitados y en la abolición de rendimientos fijos predeterminados, llamada Riba, es decir: "usura”, prohibida en el Corán (Sura 2:275):

"Los que devoran la usura se comportan como aquel a quien el toque de Satán ha sumido en el desconcierto, porque dicen: el comercio es una forma de usura, siendo así que Dios ha hecho lícito el comercio y ha prohibido la usura. Así pues, quien sea consciente de la 
advertencia de su sustentador y desista (de la usura), podrá quedarse con sus ganancias pasadas y su caso queda en manos de Dios; pero los que reincidan, jesos están destinados al fuego y en el permanecerán!".

La prohibición de Riba supone la asociación directa de cualidades morales a un hecho económico: el cobro de intereses. Con ella se condena la usura. No se considera justo que el prestamista obtenga un beneficio sin hacer frente a ningún tipo de riesgo o esfuerzo productivo.

En el Islam, toda ganancia debe estar sujeta a posibles pérdidas (a cierto componente de riesgo), o relacionada con el esfuerzo y el trabajo de los individuos, por tanto, para legitimar todo enriquecimiento se exige que se de la creación de una contrapartida en forma de trabajos, participación en el riesgo de la actividad enriquecedora, responsabilidad sobre el producto comerciado, siempre garantizando que la participación de los diferentes miembros en la actividad sea equilibrada.

En el Islam se prohíbe también la existencia de gharar. Ésta se produce cuando se dan situaciones de información imperfecta 0 asimétrica en las transacciones económicas, bien en cuanto al precio o a las características del objeto de venta en el momento de firmar un contrato o realizar una transacción. Prohibiéndolo se intenta regular las transacciones económicas para que resulten justas, honestas y equitativas. Para evitar la existencia de gharar en los contratos todas las partes deben tener acceso a toda la información sobre los objetos del contrato, precio, costes, condiciones, etc. $Y$ se prohíbe toda venta en la que el objeto no exista, esté fuera de control del vendedor, no tenga precio definido o se desconozca la fecha de su determinación.

La Banca Islámica, por tanto, se aproxima más al concepto de Banca Ética occidental en el sentido de que parte del establecimiento de unos principios morales o éticos, 
en este caso los del I slam, como base de la organización y de todas sus actividades. En concreto, la Banca Islámica se basa en los principios de la Sharia, tratando de desarrollar una práctica financiera que no contradiga las leyes islámicas.

\section{Tipos de contratos}

El banco islámico, normalmente, no concede préstamos directos. En cambio, sí puede participar en las inversiones, donde el acreedor y el deudor son participantes en el trabajo y asumen tanto las ganancias como las pérdidas. El préstamo, según la legislación islámica tiene, siguiendo a Abuamria (2006) dos posibilidades:

- La primera es la de realizar un préstamo sin interés, sin más implicaciones que el deseo de hacer el bien, con un fin humanitario o solidario.

- La segunda es la que contempla el deseo del acreedor de obtener parte del beneficio obtenido por el deudor. En este caso, tiene la opción de participar como socio, siempre aceptando la base de compartir tanto ganancias como potenciales pérdidas.

En base a esta segunda modalidad de préstamo, el sistema financiero islámico ofrece diferentes tipos de contratos que, desde el punto de vista de la legislación islámica, la Sharia, son legítimos, estos son, I qbal (2006):

A. Musharakah (asociación): Significa literalmente reparto. En la literatura de la financiación islámica se refiere a una empresa conjunta en la que todos los socios comparten los beneficios o las pérdidas de la empresa. Se refiere al acuerdo en el que dos o más partes establecen una empresa comercial conjunta y todas aportan capital, así como mano de obra y gestión, por regla general. Los beneficios de la empresa se comparten entre los socios en las proporciones acordadas, si bien las pérdidas se comparten en función de la estricta proporción de aportación de capital. 
B. Mudarabah (asociación pasiva): Es un tipo especial de asociación. Se trata de un contrato entre dos partes (aunque pueden ser más de dos), en el que un propietario de capital (llamado rabb al mal) y un gerente de inversión (llamado mudarib). El beneficio se distribuye entre las dos partes en función de la proporción que se acordara a la firma del contrato. Las pérdidas financieras las soporta el propietario del capital; las pérdidas del gerente serán el coste de oportunidad de su trabajo, ya que no se generaron ingresos.

C. Asociación en disminución: Este es un tipo de contrato entre un financiero (el banco) y un beneficiario por el que las dos partes establecen una asociación para poseer un activo, como se ha descrito con anterioridad, pero a condición de que el financiero venda gradualmente su participación al beneficiario, al precio acordado y conforme a un calendario acordado.

D. Bay al-murabahah (contrato de ventas con un margen de beneficio): En la literatura clásica, figh existe un contrato de ventas llamado bay mu'ajjal, que se refiere a la venta de bienes o propiedad contra un pago diferido (mediante una cantidad global o a plazos). El bay mu'ajjal no tiene que hacer ninguna referencia al margen del beneficio que el proveedor puede obtener. El elemento esencial que lo distingue de las ventas al contado es que el pago se aplaza. En sentido estricto, el pago aplazado puede ser superior, igual o inferior al precio al contado.

E. Ijarah (arrendamiento): En el contrato de arrendamiento sencillo, el usufructo generado con el tiempo por un activo, como por ejemplo maquinaria, aviones, barcos o trenes, se vende al arrendatario en un precio determinado. Se trata de un arrendamiento de operación, a diferencia del arriendo financiero. El arriendo de operación tiene una serie de características que lo distinguen de otras formas de arrendamiento. En primer lugar, el arrendador es el verdadero dueño del activo arrendado $y$, por tanto, soporta todos los riesgos y responsabilidades de la propiedad. En segundo lugar, el arriendo no abarca la totalidad de la vida útil del activo arrendado, sino un período específico a 
corto plazo (un mes, un trimestre 0 un año), a no ser que se renueve por consentimiento mutuo de ambas partes.

F. Arrendamiento que finaliza con la compra del activo arrendado: Dado que el arrendador soporta la totalidad del arriendo de operación, existe un riesgo de mal uso de activo arrendado por parte del arrendatario. El arriendo financiero soluciona este problema haciendo que el periodo de arriendo sea lo bastante extenso (normalmente todo el ciclo de vida útil del activo arrendado) como para permitir al arrendador amortizar el coste del activo con los beneficios. Al final del período de arriendo, el arrendatario tiene la opción de comprar el activo al arrendador a su valor actual de mercado. El arrendamiento no se puede cancelar antes del vencimiento del período de arriendo sin el consentimiento de ambas partes. Existe, por tanto, poco riesgo de un mal uso del activo.

G. Salam: Es un contrato de venta en el que el precio se paga por adelantado en el momento de la firma del contrato, contra la entrega de los bienes/servicios adquiridos en una fecha especificada. No toda la mercancía es apropiada para un contrato salam. Por lo general, se aplica únicamente a mercancías fungibles.

H. Al- istisna (contrato de fabricación) y Al-istisn Al-Tamwili (financiación a través de istisna): Al-istisna es un contrato en el que una parte solicita a otra que fabrique una determinada mercancía, cuya descripción, fecha de entrega, precio y fecha de pago se estipulan en el contrato. Cualquier parte puede cancelar el contrato una vez dado aviso a la otra antes del comienzo del trabajo de fabricación. Sin embargo, una vez que el trabajo de fabricación haya comenzado, el contrato no puede ser cancelado unilateralmente. Istisnaes similar a salam en el sentido de que ambos son excepciones a algunas condiciones generales de venta que prohíben la venta de algo que no es propiedad ni está en poder del vendedor en el momento de la venta.

I. Jualah: Se trata de un contrato para realizar una tarea determinada a cambio de unos honorarios estipulados (en un período dado). Los servicios de los 
agentes inmobiliarios son un buen ejemplo, imaginemos que alguien pone su casa en venta a través de una agencia inmobiliaria por un período de tres meses, si la agencia inmobiliaria es capaz de venderla, conseguirá una compensación estipulada, generalmente, un porcentaje del precio de la venta. Si no consigue venderla, no obtiene nada y pierde su trabajo y los gastos de publicidad. Los bancos pueden utilizar este contrato para facilitar servicios bancarios a cambio de unos honorarios fijos.

J. Wakalah: Es un contrato por el que una persona (principal) contrata a otra persona para que actúe en su nombre, por ejemplo, como su representante en una determinada tarea. El representante está autorizado a recibir unos honorarios predeterminados, independientemente de si es capaz o no de cumplir con la tares asignada a satisfacer del principal, siempre que actúe de una manera digna de confianza. Se le podría responsabilizar únicamente si se pudiese demostrar que ha incumplido los términos del contrato o que actuó deshonestamente.

K. Takaful: Una alternativa al contrato de seguros contemporáneo. Un grupo de personas acuerda compartir un cierto riesgo (por ejemplo, daños por un incendio) mediante la aportación de una suma específica individual. En el caso de pérdidas por parte de alguien del grupo, se hace frente a estas pérdidas con los fondos recaudados.

L. Sukuk: Son instrumentos para titulaciones conjuntas. Se trata de instrumentos secundarios basados en los retornos de activos reales o de su usufructo. Sukuk se destina a la movilización de recursos del mercado, basándose en la fortaleza del balance general, de las credenciales, del historial, de la buena voluntad y de las perspectivas del proyecto propuesto. Son básicamente certificados de propiedad, negociables en los mercados secundarios. En los últimos años, expertos financieros islámicos han desarrollado una serie de instrumentos. Entre los que se incluyen: ijarah sukuk, muqarradah bond/sukuk, muraba sukuk, salam sukuk, etc. 


\section{Estructura de la institución bancaria islámica}

El establecimiento de los bancos islámicos se crea a través de un contrato, a través del cual varios socios se ponen de acuerdo para aportar unas cantidades determinadas de capital a la fundación del banco. Los socios pueden ser tanto individuos como gobiernos u otras instituciones. En los bancos pequeños la toma de decisiones en la gestión del banco se crea por consenso entre lo miembros fundadores. Cuando el número de inversores aumenta, las decisiones sobre cuestiones predeterminadas las toma un Consejo de Representantes. El funcionamiento diario del banco lo lleva a cabo el Gabinete de Dirección, formado por un grupo de directores contratados.

En cuanto a la organización del banco comprende también los siguientes departamentos:

A. El Comité de supervisión religioso: aunque no lo tienen todos los bancos es el encargado de realizar el seguimiento de la actividad del banco para que no se desvíe de lo predispuesto en la Sharia. Este comité sería la figura encargada de introducir la Justicia como principio regulador de la actividad bancaria.

B. Departamento del zakat. es un impuesto islámico sobre la riqueza renta y ahorro, carga los activos y fondos ociosos con lo que recae sobre las cuentas corrientes y de ahorro que no participen en inversiones (puede representar desde el 1 al 2,5 por ciento de los depósitos. Algunos bancos se encargan de su recaudación y del uso de sus fondos hacia obras de caridad, construcción de infraestructuras, escuelas, hospitales, mezquitas, ayudas a familias 0 estudiantes, etc., según las necesidades locales y puede administrarse por el Estado u otro tipo de organizaciones. Este impuesto junto a la financiación de proyectos beneficiosos para la comunidad, constituye el principal rasgo social y redistributivo del banco. 
C. Departamento de Inversión: realiza el seguimiento de las inversiones financiadas con los fondos del banco. Se suelen especializar por áreas 0 sectores pues el éxito de su actividad depende de la información y preparación que posea respecto a los proyectos en los que se implica.

D. Departamento de Formación: se encarga de la preparación del personal en la teoría y práctica de la banca islámica además de la formación en temas de consultoría e inversiones.

Los recursos del banco provienen de los fundadores y accionistas y de los clientes depositarios, los que mantienen cuentas corrientes o de ahorro, sin remuneración y los clientes que mantienen cuentas de inversión, con participación en los beneficios del banco. Además recibe ingresos de algunos servicios que ofrece a los clientes y de la parte de beneficios que le corresponde de las actividades económicas que haya ayudado a financiar.

Y, por último, nos queda tratar el uso de los recursos bancarios que se dirige hacía la inversión del capital a través de los diferentes contratos islámicos. En estos contratos el banco participa en el riesgo de la actividad financiada, tanto en sus beneficios como en sus pérdidas. Según la modalidad de contrato también puede participar en la gestión de la actividad financiada o facilitar algún tipo de servicios relacionados con ella, por ejemplo: adquisición de materias primas, bienes inmuebles, maquinaria, distribución de productos, aportación de asesoramiento, conocimientos y contactos, etc. El porcentaje de los beneficios de las inversiones que recibe el banco depende de las características del contrato y de esta participación.

\section{Experiencia internacional de la Banca Islámica en algunos países}


Para poder comprender la situación actual de la Banca Islámica y hacer una estimación de lo que pueda suceder en el futuro hemos creído interesante analizar lo sucedido con su implantación en algunos países, por ejemplo en:

\subsection{Pakistán}

El proceso de islamización amplia del sistema bancario pakistaní se inició a continuación de una declaración del entonces presidente de Pakistán, en febrero de 1979, en la que dio a conocer que el gobierno pretendía eliminar los intereses de la economía en un período de tres años, y que se había adoptado una decisión al respecto al eliminar los intereses de las operaciones de tres instituciones financieras especializadas: House Building Finance Corporation, National Investment Trust y los fondos de inversión de Investment Corporation of Pakistan.

Para la conversión de las operaciones de los bancos comerciales en otras basadas en ausencia de intereses, se adoptó un acercamiento gradual. En principio, se adoptaron algunas medidas, en enero de 1981, para establecer mostradores separados que aceptaran depósitos basados en el reparto de beneficios/pérdidas en todas las sucursales internas de los cinco bancos comerciales nacionalizados. El sistema paralelo, en el que los ahorradores tenían la opción de colocar su dinero en los bancos, bien en depósitos con intereses o en depósitos PLS, continuó funcionando hasta finales de junio de 1985.

A partir del 1 de julio de 1985, no se permitió que ninguna sociedad bancaria aceptara ningún depósito con intereses, con la excepción de los depósitos en divisas, que siguieron obteniéndolos. Desde esta fecha, todos los depósitos aceptados por las sociedades bancarias compartieron los beneficios y las pérdidas del banco, excepto los depósitos en cuentas corrientes, a los que no correspondía ningún interés o beneficio y cuya suma de capital se garantizaba. El banco central del país dio instrucciones que especificaban los doce modos de financiación que podían emplear 
los bancos para movilizar los fondos. Sin embargo, en la práctica, los bancos confiaron casi completamente en disposiciones muraba (margen de beneficio) y de recompra.

Un primer acercamiento hacía la introducción de la técnica de financiación mudarabah se llevo a cabo en junio de 1980, con la promulgación de una ley en virtud de la cual las empresas, bancos y otras instituciones financieras podían registrarse como empresas mudarabah y movilizar los fondos a través de la emisión de certificados mudarabah. Los fondos obtenidos mediante un contrato mudarabah sólo podían ser empleados en negocios permitidos por la Sharia, y era necesaria la aprobación por parte de un Consejo Religioso que el gobierno expresamente estableció para este objetivo.

\subsection{Irán}

En 1983, se promulgó en Irán una nueva ley bancaria, la Ley de Operaciones Bancarias sin Usura. La ley requería que los bancos convirtieran sus depósitos en depósitos sin intereses en el plazo de un año, y el resto de operaciones en tres años a partir de la aprobación de la ley, especificándose los tipos de transacciones que debían constituir la base para la adquisición de activos y pasivos por parte de los bancos. La ley también especificaba las responsabilidades del Banco Central en virtud del nuevo sistema y la mecánica del control del Banco Central sobre el sistema bancario.

La ley permite a los bancos aceptar tres tipos de depósitos: depósitos gard alhasanah, depósitos generales de inversión a plazo y depósitos de inversión en proyectos específicos. Una característica importante de la Banca Islámica en Irán es que los bancos están obligados a destinar un aparte de sus recursos a la subvención de gard al-hasanah para ayudar a alcanzar los objetivos socioeconómicos establecidos en la Constitución del país. 


\subsection{Sudán}

El proceso de islamización económica del sistema bancario en este país, Iqbal(2006), no ha sido fácil ni estable. El premier intento de islamizar el conjunto del sistema bancario fue en 1984, cuando se emitió un decreto presidencial dirigido a todos los bancos comerciales para que interrumpieran todas las transacciones basadas en los intereses con efecto inmediato y negociaran la conversión de sus depósitos con intereses en formas islámicas aceptables. Se permitió, no obstante, que las transacciones extranjeras continuaran basándose en los intereses de manera temporal. Este experimento de islamización económica amplia del sistema bancario finalizó en 1985 con el cambio de gobierno. El Gobierno reavivo el proceso en 1990, reactivando una ley bancaria islámica existente. Promulgó una ley más extensa en 1992 que abarcaba la islamización económica amplia del sistema financiero, incluido el sector gubernamental. Actualmente, todos los bancos utilizan modos islámicos de financiación.

\subsection{Bahrein}

El Reino de Bahrein fue de los primeros en reconocer la importancia del concepto de banca y financiación islámicas, y como consecuencia ha respaldado el desarrollo de la industria en general y ha dado la bienvenida a las nuevas instituciones.

El primer banco islámico de Bahrein se estableció en 1979, desde entonces el sector ha crecido considerablemente. Actualmente, Bahrein cuenta con el mayor número de instituciones financieras islámicas, y no sólo en el Golfo, sino en cualquier parte del mundo, el Reino acoge 26 bancos e instituciones financieras islámicas, 5 organizaciones de respaldo industrial, 6 empresas de seguros islámicos y 34 fondos de inversión islámicos. En 2000 se introdujo por parte de la Agencia Monetaria de Bahrein (BMA) un conjunto extenso de regulaciones para los bancos, el llamado 
Marco de Información Prudencial y Reguladora (PIRI), abarcando áreas como adecuación del capital, calidad de los activos, gestión de las cuentas de inversión, gobernación corporativa y gestión de la liquidez.

\subsection{Malasia}

El sistema financiero islámico desarrollado en Malasia durante las dos últimas décadas emerge como un gran sistema financiero que opera paralelamente y es capaz de competir en el mismo terreno con el sistema financiero tradicional.

La base legal para el establecimiento de los bancos islámicos fue el Acta Bancaria Islámica (IBA) que entró en vigor el 7 de abril de 1983. Esta acta proporciona al Banco Negara Malasia (BNM) poderes para supervisar y regular los bancos islámicos, de forma similar a otros bancos autorizados. El Acta de Inversión del Gobierno, de 1983, fue también promulgada con el fin de autorizar al Gobierno de Malasia a emitir Certificados de Inversión del Gobierno (GIC), que son valores emitidos por el Gobierno basados en los principios de la Sharia.

El primer banco islámico establecido en el país fue el Bank Islam Malaysia Berhad (BIMB) que comenzó sus operaciones en 1983. En 1999 comenzó sus operaciones el Bank Muamalat Malaysia Berhad (BMMB). Actualmente, más de veinte bancos ofrecen servicios bancarios islámicos, además existen más de cincuenta fondos de inversión islámicos.

\subsection{Arabia Saudí}

Es el principal mercado financiero islámico en términos de tamaño, el mayor banco islámico del mundo, Al Rajhi Banking and Investment Corporation, tiene su sede en este país. También supone la mayor concentración de fondos islámicos y es la sede oficial del Banco Islámico del Desarrollo. 
El Banco Islámico del Desarrollo es un banco de desarrollo multilateral que da servicio a los países musulmanes. Sus socios actuales se encuentran en 57 países. Su objetivo es fomentar el desarrollo económico y el progreso social de los países miembros y de las comunidades musulmanas, individual y colectivamente, de acuerdo con los principios de la Sharia. Con el fin de satisfacer las necesidades crecientes y diversas de sus países miembros, el Banco ha establecido varias instituciones y fondos con disposiciones administrativas y reglas operacionales distintas. Estas entidades y fondos afiliados del Banco permiten al BID movilizar recursos financieros suplementarios, en línea con los principios de la Sharia y centrarse en aquellas funciones y actividades que no pueden ser cubiertas bajo las disposiciones normales de financiación. Con estas entidades y fondos afiliados, el Banco ha evolucionado con el tiempo hasta convertirse en un grupo llamado Grupo del BID.

\section{Situación actual y retos}

En mayo de 2006, en Amman (Jordania), tuvo lugar el quinto encuentro del Islamic Financial Services Board, institución creada en 2002 para supervisar la industria de las finanzas islámicas y de la que son miembros tanto el Banco Islámico de Desarrollo, como el Banco Mundial o el FMI. En el encuentro se discutieron los retos de la globalización financiera para las finanzas islámicas. Londres, acogió en Junio, la cumbre sobre bonos islámicos, Sukuk Summit 2008 y en julio, el primer congreso en Europa de la Conferencia Mundial Anual de Banca Islámica, normalmente celebrado en Bahrein. En Reino Unido, como en países de Oriente Medio y el sudeste asiático, se suceden las conferencias y congresos sobre financiación, seguros y mercados de capital islámicos.

Para Orozco (2008), la industria de la financiación islámica está sin duda, en expansión. Con tasas de crecimiento de activos entre el 10 y el 15 por ciento, en los 
últimos años, se espera que crezca en 2008 un 20 por ciento. En total, 300 instituciones financieras islámicas operan en distintos países del mundo, cifra que se ha casi duplicado en los últimos doce años (en 1996, el directorio de la Asociación Internacional de Bancos Islámicos, el IAIB, recogía un total de 166 instituciones financieras, 192 si se incluían las que no eran miembros de dicha asociación). Las cifras de negocio que mueven, oscilan en torno a los 300 y 500.000 millones de dólares en activos, según estimaciones de Munawar Iqbal y del FMI, algunas de las cuales predicen que alcancen el billón de dólares en los próximos dos años.

Los bancos islámicos más grandes se encuentran en el Golfo, hasta ahora el Al-Rajhi Bank y la Kuwait Finance House. Los bancos más dinámicos y competitivos se encuentran en el sudeste asiáticos, aunque siguen representando sólo un nicho de mercado financiero en estos países.

Resulta inevitable unir este nuevo auge que viven las finanzas islámicas al de las rentas del petróleo. Si bien, como hemos comentado con anterioridad, los primeros bancos islámicos aparecen en los años setenta del siglo pasado, es con el primer boom petrolífero cuando la industria comienza su expansión. En estos últimos años, la tendencia alcista de los precios del petróleo, ha permitido que parte de los excedentes de liquidez generados en Oriente Medio se canalice de nuevo hacía los bancos islámicos, que se están expandiendo más allá de la región o el sudeste asiático hacía el Magreb y Europa.

Tras entrar en los mercados de Túnez y Siria en 2005, Marruecos permitió el uso y oferta de productos financieros "alternativos" en octubre de 2007. En Europa, Reino Unido, con una población musulmana de origen mayoritariamente pakistaní, pionera en las finanzas islámicas, se crearon en 2004 y 2006 los dos primeros bancos comerciales de carácter islámico de Europa (Islamic Bank of Britan y European Islamic Investment Bank). Muchas otras instituciones financieras europeas, entre ellas ABM Amor, Banque Nacional du Paris, Deutsche Bank, HSBC, Lloyds TSB y la 
Unión de Bancos Suios, Ubs, entre otros, están comenzando o ofrecer productos financieros, hipotecas, cuentas corrientes y bonos islámicos (sukuk). Otros grupos financieros europeos participan también en bancos islámicos del golfo, Pakistan o Malasia en la emisión de bonos islámicos 0 en diversas transacciones financieras islámicas, como Barclays Bank, Standard Chartered Bank y, de nuevo, Deutsche Bank y UBS.

Tanto Reino Unido como Francia, han modificado recientemente sus códigos fiscales para reducir la carga impositiva que sufren tanto bonos como hipotecas islámicas, al declarar los rendimientos como renta y no como interés.

En España, y teniendo en cuenta que ya viven en nuestro país un millón y medio de musulmanes, la Junta Islámica y diferentes instituciones financieras, están estudiando, desde hace algunos años, la posibilidad de crear un banco islámico 0, en su defecto, cuentas corrientes "halal". Hasta ahora los intentos han sido fallidos pero todo parece indicar que en un futuro inmediato se llegarán a acuerdos, sobre todo si tenemos en cuenta lo sucedido en nuestros vecinos y socios europeos.

La Banca Islámica, como cualquier otro sistema bancario, debe contemplarse como un sistema en evolución. Desde sus inicios modestos en los años 70 , como hemos visto, ha mostrado un gran progreso en los últimos treinta años, estableciéndose en entornos heterogéneos sociales y económicos.

La Banca Islámica cumple los requisitos religiosos de los musulmanes pero también amplía la gama de opciones disponibles a otras personas, al ofrecer tanto financiación de ventas como productos de bajo riesgo (por ejemplo, compra y venta) y productos basados en el reparto de riesgos y beneficios. Además de ofrecer más opciones a los clientes, esta combinación de modos de retorno de beneficios fijos y variables tiene una serie de efectos beneficiosos para la eficiencia y la estabilidad del sistema. La Banca islámica no debería considerarse sólo como un movimiento 
religioso. Se trata simplemente de otra forma de realizar la función de intermediación financiera.

\section{Bibliografía}

ABUAMRIA, F. (2006): "La banca islámica en el mundo: principales rasgos y análisis de eficiencia" en Cuadernos de Información Económica, no 193, págs. 87-94.

ALSINA, O. (2002): La banca ética. Mucho más que dinero. Ed. I caria Milenrama. Barcelona. BALLESTEROS, C. (2003): "La banca ética" en SICHAR, G. (Coord): La empresa socialmente responsable. Ed. CIDEAL, págs.29-123.

CLAVERO, B. (1984): Usura: del uso económico de la Religión en la Historia. Ed. Tecnos. Madrid.

DE ANCA, C. (2008): "Invertir en valores: inversión socialmente responsable e inversión islámica" en Boletín de Economía y Negocios, oo 6. Ed. Casa Árabe.

DE LA CUESTA, M. y DEL RíO, N. (2001): "Dinero más ético y solidario para una sociedad más humana y responsable" en Noticias de economía política social y cooperativa, № 33, julio 2001, págs. 46-52.

DE LA CUESTA, M., FERNÁNDEZ, B. y VÁZQUEZ, O. (2006): Razón de ser de la Banca ética: experiencias internacionales y nacionales. FUNCA. Madrid.

DEL RIO, N. (2003): Rescata tu dinero. Finanzas solidarias y transformación social. Ed. TALASA. Madrid.

IQBAL, M. (2006): "Banca islámica: teoría, práctica y evolución" en Papeles de Economía Española, no 110, págs. 166-183.

KHAN, M. y MIRAKHOR, A. (1986): "Estructura y práctica de la banca islámica" en Finanzas y Desarrollo, № 23, págs. 32-36.

LORCA, A. y OROZCO, O. (1999): La banca islámica sin intereses: elementos básicos. Agencia Española de Cooperación Internacional. Madrid. 
MONTURIOL, Y. (2006): Términos clave del Islam. Ed. J unta I slámica. Córdoba.

OROZCO, O. (2008): "La Banca Islámica: nuevo auge y expansión en el Magreb y Europa" en Boletín de Economía y Negocios, no 6. Ed. Casa Árabe.

PRADO, A. y COLLADO, J. (2008): "Islam y democracia" (Manual del modulo V). Curso de experto en Cultura y Religión islámicas. UNED. Madrid.

SAN JOSÉ, L. y RETOLAZA, J. L. (2006): “Análisis comparativo de la Banca Ética con la Banca Tradicional: Identificación de indicadores" en Conocimiento, innovación y emprendedores: camino al futuro. Ed. Ayala Calvo, J. C., Diciembre, págs. 1104-1118.

SASIA, P.M. y DE LA CRUZ, C. Banca Ética y Ciudadanía. Trotta. Madrid.

\section{Webgrafía}

Banca Ética. Disponible en: www.bancaetica.com. Consultado el 01-06-2006.

Banco Santander. Disponible en: www.bancosantander.com. Consultado el 07-06-2006. $B B V A$. Disponible en: www.bbva.es. Consultado el 12-05-2006

$B N E$. Disponible en: www.bne.es. Consultado el 12-06-2006.

CASA ÁRABE. Disponible en: www.casaarabe.com. Consultado el 17-05-2006.

CS/C. Disponible en: www.csic.es. Consultado el 25-05-2006.

FIARE. Disponible en: www.fiare.org. Consultado el 15-06-2006.

ISDB. Disponible en: www.isdb.org. Consultado el 18-06-2006.

OCl-OCl. Disponible en: www.oic-oci.org. Consultado el 15-06-2006.

TRIODOS. Disponible en: www.triodos.es. Consultado 22-06-2006.

UCM. Disponible en: www.ucm.es. Consultado el 01-06-2006.

WEB ISLAM. Disponible en: www.webislam.com. Consultado el 02-06-2006. 